\title{
Molecular Regulation of the Mitosis/Meiosis Decision in Multicellular Organisms
}

\author{
Judith Kimble \\ Howard Hughes Medical Institute, Department of Biochemistry, University of Wisconsin-Madison, \\ Madison, Wisconsin 53706 \\ Correspondence: jekimble@wisc.edu
}

\begin{abstract}
A major step in the journey from germline stem cell to differentiated gamete is the decision to leave the mitotic cell cycle and begin progression through the meiotic cell cycle. Over the past decade, molecular regulators of the mitosis/meiosis decision have been discovered in most of the major model multicellular organisms. Historically, the mitosis/meiosis decision has been closely linked with controls of germline self-renewal and the sperm/ egg decision, especially in nematodes and mice. Molecular explanations of those linkages clarify our understanding of this fundamental germ cell decision, and unifying themes have begun to emerge. Although the complete circuitry of the decision is not known in any organism, the recent advances promise to impact key issues in human reproduction and agriculture.
\end{abstract}

\begin{abstract}
Cerm cells face a number of major fate $\checkmark$ decisions during their development. One is self-renewal or differentiation; another is how to differentiate as a gamete of a particular sex, in animal sperm or egg; and a third is a cell cycle decision-to leave the mitotic cell cycle and enter the meiotic cell cycle, or more simply, the mitosis/meiosis decision. A longstanding puzzle in germ cell biology has been to understand the regulatory relationships among these three fate decisions. How are germ cells controlled to leave their stem cell mode in the mitotic cell cycle and then enter the meiotic cell cycle and mature as sperm or egg? Our understanding of this complex process comes largely from model animals - the nematode Caenorhabditis elegans, fruit fly Drosophila melanogaster, and mouse Mus musculus, as well as
\end{abstract}

model plants-the dicot Arabidopsis thaliana and monocots maize Zea mays and rice Oryza sativa. Although the regulatory circuitry remains fragmentary in virtually all these multicellular organisms, unifying themes have begun to emerge. The major advances made over the past decade have answered central questions and posed new or clearer questions that will likely shape the field in the coming few years.

\section{GENERAL FEATURES OF MITOSIS, MEIOSIS, AND THE MITOSIS/MEIOSIS TRANSITION}

Certain features of the mitotic and meiotic cell cycles are universal. During the mitotic cell cycle, the genome is replicated during S-phase, the duplicated chromosomes segregate from each other during $\mathrm{M}$-phase and then become

Editors: Paolo Sassone-Corsi, Margaret T. Fuller, and Robert Braun

Additional Perspectives on Germ Cells available at www.cshperspectives.org

Copyright (C) 2011 Cold Spring Harbor Laboratory Press; all rights reserved; doi: 10.1101/cshperspect.a002683

Cite this article as Cold Spring Harb Perspect Biol 2011;3:a002683 
J. Kimble

incorporated into two diploid daughters. S-phase is preceded by $\mathrm{G}_{1}$, a stage when key developmental controls are exerted, and it is followed by $\mathrm{G}_{2}$, a stage characterized by growth and preparation for M-phase. In animals, primordial germ cells (PGCs) proliferate using the mitotic cell cycle in embryos, and adult germline stem cells (GSCs) rely on the mitotic cycle for self-renewal and amplification via trans-amplifying cells.

As germ cells begin to mature, they transition from the mitotic cell cycle into the meiotic cell cycle, a process that ultimately reduces the number of chromosomes from two sets to one in both sperm and egg (Fig. 1). Fertilization restores the two sets of chromosomes in the newly formed zygote, which then launches a new generation. The meiotic cell cycle begins with a "meiotic S-phase" during which the genome is replicated and a meiosis-specific cohesin complex is loaded onto chromosomes. This meiotic S-phase constitutes a first and crucial step in the meiotic cell cycle (e.g., Watanabe et al. 2001; Forsburg 2002). After meiotic S-phase, germ cells enter an elaborate and protracted meiotic prophase I, during which sister chromatid pairing, synapsis, and recombination occur. The first easily visible hallmarks of the meiotic cell cycle are chromosome pairing and early steps in formation of the synaptonemal complex during leptotene and zygotene. These early meiosis-specific events are often detected by their characteristic nuclear morphology and by immunocytochemistry using antibodies against early meiotic prophase proteins (e.g., components of the synaptonemal complex).

\section{MOLECULAR REGULATION OF THE MITOSIS/MEIOSIS DECISION IN SPECIFIC ORGANISMS}

Our understanding of the mitosis/meiosis decision in multicellular creatures comes largely from focused studies in model organisms with each bringing its own perspective to bear on the mitosis/meiosis decision. Although many aspects of the mitotic and meiotic cell cycles are conserved during germ cell development, the transition from one to the other often takes place in an organism-specific or sex-specific context. As a result, the mitosis/meiosis decision is presented to the experimentalist in ways that have impacted how it has been approached in different organisms. We begin this section with a very brief summary of insights from single-celled yeasts, and then turn to the decision in multicellular organisms, emphasizing recent advances at the molecular level.

\section{THE YEASTS: DIVERSE MOLECULAR MECHANISMS OF MITOSIS/MEIOSIS REGULATION}

Elaborate regulatory circuitries control the mitosis/meiosis decisions in the budding yeast Saccharomyces cerevisiae and the fission yeast
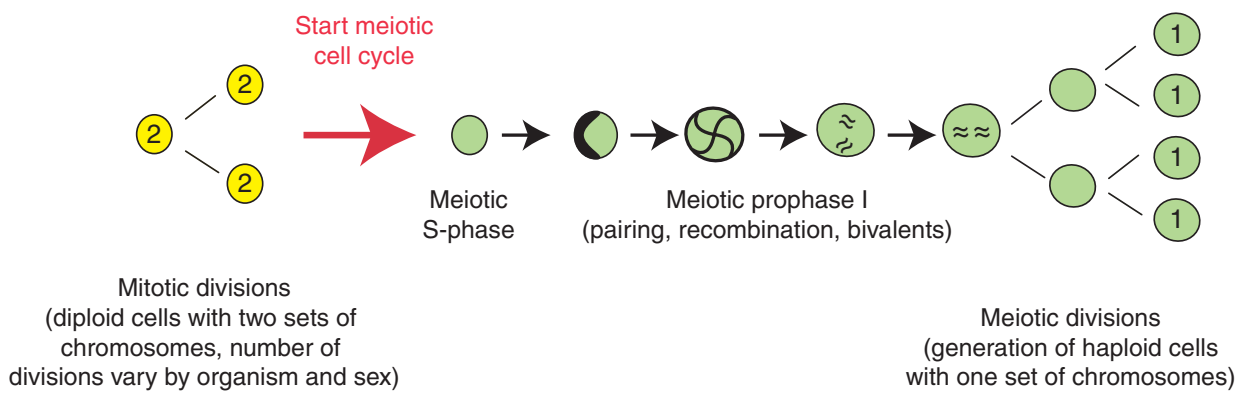

Figure 1. Universal features of mitotic and meiotic germ cell cycles. Mitotic divisions duplicate diploid germ cells (yellow cells, 2 depicts diploidy), whereas meiotic divisions generate haploid germ cells (green cells, 1 depicts haploidy). The red arrow shows that the decision to leave the mitotic cell cycle and enter the meiotic cell cycle occurs prior to meiotic S-phase. Meiotic prophase I progresses from its earliest stages (crescent depicts chromosomal bouquet), through pachytene (wavy lines depict synapsed chromosomes), to later stages (chromosomal bivalents) that line up at the metaphase plate at meiosis division I. 
Schizosaccharomyces pombe. Genes regulating the mitosis/meiosis decision were initially recognized in mutants that continue mitotic divisions under conditions that normally signal meiotic entry, or conversely in mutants that aberrantly enter the meiotic cycle when mitotic divisions are the norm. A common theme is that the nutritional environment combines with sex determination regulators to ensure that yeast cells enter the meiotic cell cycle only when they are in a diploid state and find themselves without sufficient nutrition.

\section{S. cerevisiae}

The mitosis/meiosis decision is arguably best understood in the budding yeast, $S$. cerevisiae. The IME1 transcription factor acts in late $G_{1}$ or at the $G_{1} / S$ transition to activate the transcription of genes in the early meiotic genetic program and to initiate meiotic S-phase (Mitchell 1994; Vershon and Pierce 2000; Kassir et al. 2003). The regulation of IME1 is accomplished by nutritional cues and other inputs. For example, only diploid cells enter the meiotic cell cycle, and it is two key sex determination regulators (known in yeasts as mating type regulators) that register the diploid state: a1/ $\alpha 2$ represses an ime1 repressor and thereby activates the meiotic program. Manipulation of nutritional cues reveals that the mitosis/meiosis decision must be maintained. Thus, starvation of diploid cells induces entry into the meiotic cell cycle, but a return to rich medium can reverse the decision and return the cell to the mitotic cell cycle; indeed "commitment" to progression through meiotic prophase I can be reversed until the first meiotic division (Simchen 2009). See reviews for additional information about this paradigm of regulatory circuitry (Mitchell 1994; Kassir et al. 2003).

\section{S. pombe}

The mitosis/meiosis decision in the fission yeast, $S$. pombe, also relies on nutrition and sex determination, but in this case, the terminal regulators of meiotic entry are not transcription factors. Instead, nutritional cues and mating-pheromone signaling trigger an elaborate cascade that ultimately controls activity of the Mei2 RNA-binding protein. Mei2 works together with the noncoding meiRNA to initiate meiotic S-phase and also to promote subsequent events in early meiotic prophase I (Watanabe and Yamamoto 1994). Whereas meiotic mRNAs are eliminated during the mitotic cell cycle, Mei2 and meiRNA antagonize that elimination and induce meiotic entry (Harigaya et al. 2006). See reviews for additional information about this intriguing mechanism of mitosis/meiosis control (Yamamoto 1996; Harigaya and Yamamoto 2007).

\section{ELEGANS: STEM CELL CONTROLS MEET THE MITOSIS/MEIOSIS DECISION}

The C. elegans mitosis/meiosis circuitry is closely linked to controls of germline self-renewal and differentiation. As in yeast, nematode regulators of the mitosis/meiosis decision were identified in mutants that transform mitotic germ cells into the meiotic cell cycle or vice versa. These C. elegans transformations dramatically affect germline self-renewal and differentiation in addition to their effects on the mitosis/meiosis decision. When germ cells are driven aberrantly from the mitotic into the meiotic cell cycle, germline stem cells (GSCs) are lost, and conversely when germ cells aberrantly continue the mitotic cell cycle at the expense of meiotic entry, a germline tumor results.

\section{Background to C. elegans GSCs and Mitosis/Meiosis Decision}

C. elegans can develop as either an XX selffertilizing hermaphrodite or an XO male. In both sexes, GSCs proliferate from two to $\sim 1000$ germ cells during larval development (Kimble and White 1981); they maintain a constant number of germ cells in adults, while at the same time generating a steady stream of gametes (Crittenden et al. 2006; Morgan et al. 2010). A stem cell niche of somatic origin resides at the distal end of the germline tissue and is responsible for both larval GSC proliferation and adult GSC self-renewal (Kimble and 
J. Kimble

White 1981; Morgan et al. 2010). In adults, 35-70 mitotic germ cells at the distal-most end are maintained in an essentially equivalent stem cell-like state; more proximally, transitamplifying germ cells remain in the mitotic cell cycle but are organized in a distal-toproximal gradient of increasing maturation (Fig. 2A) (Cinquin et al. 2010). Therefore, the meiotic program is triggered before germ cells leave the mitotic cell cycle and overtly enter meiotic S-phase. Even more proximally, all germ cells have entered meiotic S-phase or progressed into early stages of meiotic prophase
I (Fig. 2A). When late larvae are starved, they metabolize most of their germline tissue, with only $\sim 35$ GSCs remaining to replenish the entire tissue and generate progeny after refeeding during adulthood (Angelo and Van Gilst 2009). The molecular regulation of GSCs and the mitosis/meiosis decision have been analyzed in well-fed animals, but this recent result shows unequivocally that nutritional cues have a major impact. Consistent with this idea, insulin signaling can affect the extent of larval GSC proliferation (Michaelson et al. 2010).

\section{A}
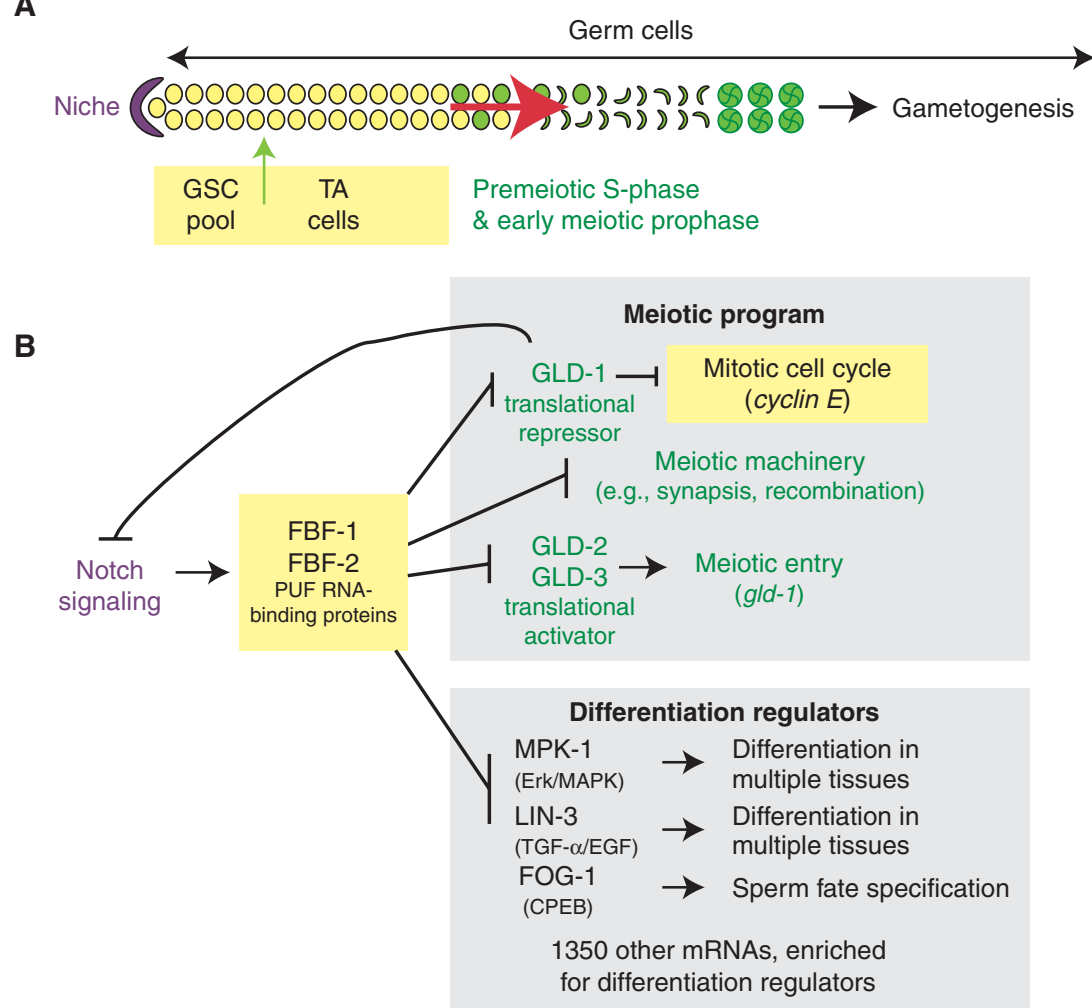

Figure 2. The C. elegans mitosis/meiosis decision. (A) The nematode germline possesses a GSC pool within its somatic niche. Germ cells in the mitotic cell cycle (yellow) extend beyond the niche and include transitamplifying (TA) germ cells, which have been triggered to begin maturation toward meiotic entry (green arrow); germ cells in the meiotic cell cycle (green) are more proximal. Overt entry into the meiotic cell cycle (red arrow) occurs asynchronously. Conventions are as in Figure 1. (B) Molecular regulation of the C. elegans mitosis/ meiosis decision includes Notch signaling from the niche and FBF maintenance of GSCs, including repression of the meiotic program. Arrows indicate positive regulation; barred lines indicate negative regulation. Solid arrows and lines indicate direct molecular regulation: Notch signaling directly activates $f b f-2$ transcription and FBF directly represses mRNAs of the meiotic program as well as key differentiation regulators. 
Regulators that Promote Germline Self-Renewal Directly Repress

the Meiotic Program

Notch signaling and two PUF ( for Pumilio and FBF) RNA-binding proteins maintain germline self-renewal and also promote continued mitotic divisions at the expense of meiotic entry (Fig. 2B) (e.g., Kimble and Crittenden 2007). A Notch ligand from the niche keeps GSCs in the mitotic cell cycle and in a stem cell-like state. When signaled, the GLP-1/Notch receptor triggers transcriptional activation of target genes (Petcherski and Kimble 2000). Two GLP-1/ Notch target genes relevant to the mitosis/ meiosis decision are $f b f-2$ (Lamont et al. 2004) and lip-1 (Lee et al. 2006). FBF-2 is discussed below. LIP-1 is the homolog of a dual specificity phosphatase that normally delays meiotic entry, probably by inactivating MPK-1, the single C. elegans homolog of ERK/MAP kinase (Berset et al. 2001; Lee et al. 2006). Other targets of Notch transcriptional activation must exist, because an $f b f$ - 2 lip-1 double-mutant maintains GSCs and supports a virtually normal mitosis/ meiosis decision. Importantly, both Notch signaling and FBF control GSCs and the mitosis/ meiosis decision in both sexes during adulthood-when XX germlines are oogenic and $\mathrm{XO}$ germlines are spermatogenic.

Two nearly identical PUF proteins, FBF-1 and FBF-2 (collectively known as FBF), function within germ cells to regulate both selfrenewal and the mitosis/meiosis decision (Fig. 2B) (e.g., Kimble and Crittenden 2007). FBF prevents meiotic entry by directly repressing the expression of a battery of mRNAs critical to the meiotic program, including regulators of meiotic entry ( $g l d$ mRNAs, see below) as well as components of the meiotic machinery (e.g., synaptonemal complex formation, recombination) (Crittenden et al. 2002; Eckmann et al. 2004; Merritt et al. 2008; Kershner and Kimble 2010; Merritt and Seydoux 2010). In addition to its direct control of the meiotic program, FBF also represses mpk-1 mRNA, lin-3 mRNA, and fog-1 mRNA, which encode the C. elegans homologs of ERK/MAP kinase, TGF- $\alpha /$ EGF, and CPEB, respectively (Fig. 2B)
(Thompson et al. 2005, 2006; Lee et al. 2007a; Kershner and Kimble 2010). MPK-1 and LIN3 regulate multiple cell fates in somatic tissues (Sundaram 2006); MPK-1 also affects germ cell fate specification (Lee et al. 2007b); FOG1 is one of the terminal regulators of sperm fate specification (Barton and Kimble 1990). Indeed, FBF has at least 1350 likely targets, many of which encode potent regulators of differentiation (Kershner and Kimble 2010). Therefore, FBF appears to maintain GSCs in a naïve stem cell-like state by repressing a broad spectrum of differentiation regulators, including those in the meiotic program.

\section{Regulators that Promote Meiotic Entry Oppose Germline Self-Renewal and the Mitotic Cell Cycle}

Three GLD proteins drive germ cells into the meiotic cell cycle (Fig. 2B) (Kadyk and Kimble 1998; Eckmann et al. 2004; Hansen et al. 2004). These GLD proteins form two branches of control, with GLD-1 in one branch and GLD-2/GLD-3 in the other branch. Importantly, the two GLD branches control meiotic entry in both XX and XO animals (Kadyk and Kimble 1998); therefore, regulation of meiotic entry appears to be gender-neutral.

GLD-1 is a translational repressor (Lee and Schedl 2010) that controls regulators of both self-renewal and the mitotic cell cycle. Its direct targets include mRNAs encoding the GLP-1/ Notch receptor and cyclin E (Marin and Evans 2003; Biedermann et al. 2009). GLD-1 also maintains commitment to progression through the meiotic cell cycle; germ cells revert to mitotic divisions and form a germline tumor in gld-1 single mutants (Francis et al. 1995; Subramaniam and Seydoux 2003; Biedermann et al. 2009); indeed within that tumor, germ cells can trans-differentiate into a variety of somatic cell types (Ciosk et al. 2006). Therefore, GLD-1 promotes meiotic entry and also maintains germ cells in a totipotent state during meiotic progression.

The GLD-2/GLD-3 heterodimer is a cytoplasmic poly(A) polymerase and translational activator (Wang et al. 2002; Suh et al. 2006). 
J. Kimble

One direct target of GLD-2/GLD-3 activation is the gld-1 mRNA, a regulatory link that provides positive feed-forward to drive meiotic entry (Suh et al. 2006); other direct targets are not yet known but must exist because GLD-2/ GLD-3 promotes meiotic entry in gld-1 null mutants. Like the GLD-1 regulator, GLD-2 and GLD-3 also govern meiotic progression, but their roles are distinct (Kadyk and Kimble 1998; Eckmann et al. 2002).

Shifting the Balance between Mitotic and Meiotic Modes of the Network

The FBF and GLD proteins form major hubs in the network of mitosis/meiosis control and the nature of those hubs confers plasticity on the decision (Kimble and Crittenden 2007). Feedback within the network is provided by GLD1 repression of Notch signaling (Marin and Evans 2003) and FBF negative auto- and crossregulation (Lamont et al. 2004). When either FBF-1 or FBF-2 is removed, the other FBF is up-regulated and GSCs are maintained, but when both are removed, GSCs are lost. Similarly, when only one GLD branch is removed, meiotic entry occurs, albeit abnormally, but when both branches are removed a germline tumor results. Intriguingly, the individual FBFs affect the balance between mitotic and meiotic modes differently, probably because of the gene-specific regulation of the two proteins (Crittenden et al. 2002; Lamont et al. 2004). In addition to the FBFs and GLDs, other regulators also shift the number of mitotically dividing germ cells (e.g., LIP-1, see above). This redundancy and modulation renders the mitosis/meiosis decision both robust and plastic, features that permit its regulatory network to respond to varying inputs (e.g., gender, aging, stress, pathogenesis, natural selection).

Many other regulatory proteins influence GSC mitotic divisions and the mitosis/meiosis balance (Kimble and Crittenden 2005; van den Heuvel 2005). The canonical cell cycle machinery controls GSC mitotic divisions, as expected; in addition, many RNA regulatory proteins affect the mitosis/meiosis balance, underscoring the importance of posttranscriptional controls of
C. elegans germ cell cycles (e.g., Belfiore et al. 2004; Ciosk et al. 2004; Eckmann et al. 2004; Maine et al. 2004; Hansen et al. 2005; Thompson et al. 2005; Hasegawa et al. 2006; Ariz et al. 2009; Kerins et al. 2010). In addition, micro RNAs have been implicated in GSC proliferation controls (Cox et al. 1998; Smardon et al. 2000). Elucidation of the complete molecular network controlling the C. elegans mitosis/meiosis decision is clearly a work in progress.

\section{DROSOPHILA: CYST FORMATION MEETS THE MITOSIS-MEIOSIS DECISION}

The Drosophila germline tissue is organized with stem cells at one end and differentiating gametes at the other (Fig. 3A, male germline). Although male and female germlines differ in many respects, some striking similarities do exist. In both sexes, GSCs divide asymmetrically to produce one GSC daughter and one daughter destined to differentiate; the latter is called a "cystoblast" in females and a "gonialblast" in males. The cystoblast/gonialblast then generates a 16-cell cyst in both sexes. In males, meiotic S-phase occurs in the germ cells of the 16 -cell cyst and their meiotic divisions generate 64 sperm. In females, two of the germ cells in the 16-cell cyst enter the meiotic cell cycle and one of those goes on to generate a single egg; the 15 others become nurse cells. Regulation of the GSC-cystoblast/gonialblast decision has been elucidated in exquisite detail in both males and females (see Davies and Fuller 2008; Zhang and Xie 2009). Importantly, two key regulators of that early decision also control meiotic entry in the germ cells of the 16-cell cyst and appear to do so in both sexes.

The bag-of-marbles (bam) and benign gonial cell neoplasm $(\mathrm{bgcn})$ proteins function in both males and females to promote "postcyst" differentiation, including meiotic entry (Fig. 3B) (McKearin and Spradling 1990; Gönczy et al. 1997; Insco et al. 2009). Germ cells lacking either bam or $b g c n$ undergo extra rounds of mitotic division and fail to enter the meiotic cell cycle. Moreover, GSCs exposed to ectopic Bam protein are induced to differentiate (Ohlstein and McKearin 1997). The Bam molecular 
A

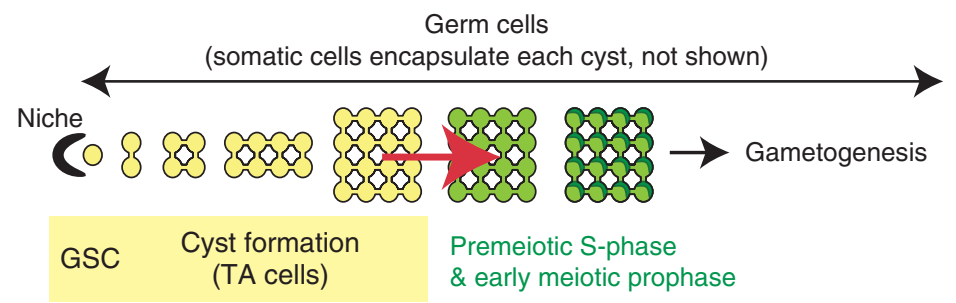

B

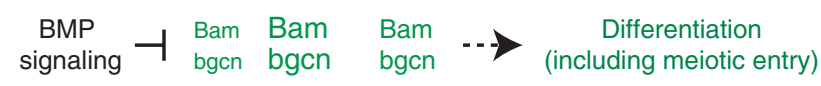

Figure 3. The Drosophila mitosis/meiosis decision. (A) The Drosophila germline possesses asymmetrically dividing GSCs within its somatic niche; transit-amplifying divisions form a 16-cell cyst and meiotic entry (red arrow) occurs in that 16-cell cyst. In males, all 16 germ cells enter the meiotic cell cycle, as depicted here; in females, only two of the 16 germ cells enter the meiotic cell cycle (not shown). Conventions and acronyms are as in Figures 1 and 2. (B) Molecular regulation of the Drosophila mitosis/meiosis decision relies on extrinsic BMP signaling and intrinsic Bam/bgen RNA regulation, with abundant Bam controlling the position of meiotic entry. Solid arrows and lines indicate direct molecular regulation; dashed line indicates control that may be either direct or indirect.

function was not apparent from its amino acid sequence (McKearin and Spradling 1990), but bgen is related to DExH proteins, suggesting a role in RNA regulation (Ohlstein et al. 2000). Indeed Bam and bgen form a complex that binds and antagonizes both Pumilio and eIF4A proteins and that also regulates the Nanos mRNA via its 3'UTR (Li et al. 2009; Shen et al. 2009; Kim et al. 2010). Therefore, Bam/bgen functions posttranscriptionally.

The transition from mitotic germ cell divisions within the cyst into meiotic S-phase normally occurs at the 16-cell stage. In Drosophila testes, Bam is normally expressed in 4-, 8-, and 16-cell cysts; its levels peak in 8-cell cysts, continue at a lower level in early 16-cell cysts that are in meiotic S-phase, and drop precipitously in later 16-cell cysts that have completed DNA replication (Insco et al. 2009). Manipulations of Bam dosage dramatically affect the timing of meiotic entry. In bam/ + heterozygotes, Bam peaks later than normal and the switch into meiosis occurs late, resulting in 32-cell and >32-cell cysts; by contrast, a PEST-deleted mutant BAM peaks earlier than normal and the switch into meiosis occurs early, resulting in 8 -cell cysts that go on to differentiate. Therefore, in Drosophila testes, the amount of Bam protein is crucial for determining the time at which cyst cells stop dividing mitotically and instead begin meiotic S-phase. Indeed, normal cyst formation relies on the Bam gradient extending between GSC and the site of meiotic entry (Fig. 3B) (Insco et al. 2009).

BMP signaling down-regulates Bam expression in both male and female GSCs, and that down-regulation is crucial for GSC maintenance (Fig. 3B) (Chen and McKearin 2003; Kawase et al. 2004). Taking these results together, the Drosophila mitosis/meiosis decision appears to be integrated with controls of GSC self-renewal maintenance, much as it is in C. elegans. In addition to the few regulators mentioned here, which interface clearly with the mitosis/meiosis decision, many other regulators affect Drosophila GSC self-renewal and differentiation and therefore are implicated in the mitosis/meiosis decision.

\section{MOUSE: SEX DETERMINATION MEETS THE MITOSIS/MEIOSIS DECISION}

The murine mitosis/meiosis decision shows a dramatic sex-specific timing of meiotic entry that historically has been analyzed as a sex determination decision. The sex-specific difference 
J. Kimble

occurs soon after the primordial germ cells (PGCs) migrate into the fetal gonadal primordium, when all female PGCs enter the meiotic cell cycle and all male PGCs arrest at $\mathrm{G}_{1} / \mathrm{G}_{0}$ of the mitotic cell cycle (Fig. 4A, left). The female germ cells quickly progress through meiotic prophase I and arrest at metaphase of the first meiotic division, still within the fetal ovary; then after puberty, those female germ cells develop in cyclical fashion to produce mature oocytes. By contrast, male germ cells at puberty take on the dual task of maintaining spermatogonial stem cells and generating a continuous stream of sperm (Fig. 4A, right). This striking sexspecific meiotic entry led to a major question in the field: Are mitosis/meiosis and sperm/ oocyte two decisions or only a single decision (Kimble and Page 2007)? An answer to that question has now emerged through groundbreaking studies that have outlined central players in the murine mitosis/meiosis pathway.

\section{Retinoic Acid and STRA8 Promote Meiotic Entry}

Success in the hunt for murine regulators of the mitosis/meiosis decision began with two key discoveries. A target gene of retinoic acid signaling, STRA8 (stimulated by retinoic acid 8), was expressed sex-specifically in female fetal gonads at the time of female-specific meiotic entry (Oulad-Abdelghani et al. 1996; Koubova et al. 2006), and the Cyp26b1 inhibitor of retinoic acid signaling was expressed sex-specifically in male fetal gonads at the same time (Bowles et al. 2006). Both findings led investigators to the idea that retinoic acid (RA) might induce meiotic entry in the fetal ovary, whereas

A
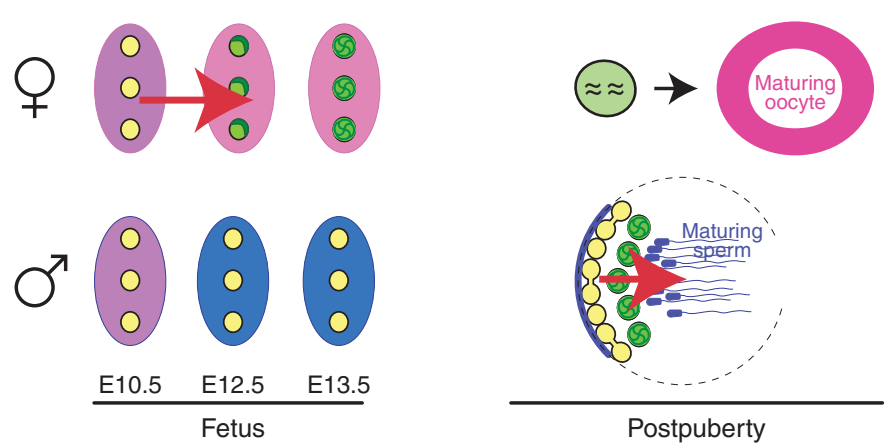

B

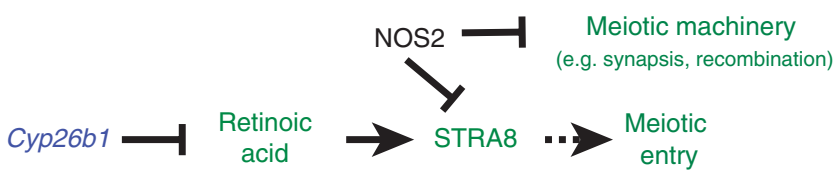

Figure 4. The mouse mitosis/meiosis decision. (A) Meiotic entry (red arrow) occurs during fetal development in female mice and after puberty in males. PGCs (yellow) enter the fetal somatic gonad, which is sexually bipotential (purple). Sex determination of the fetal somatic gonad results in a female (rose) or male (blue) somatic gonad; in these fetal developing gonads, female PGCs enter the meiotic cell cycle (green), whereas male PGCs arrest in the mitotic cell cycle (yellow). After puberty, female germ cells produce mature oocytes, whereas the male maintain spermatogonial stem cells (yellow), transit-amplifying cells (yellow) and meiotic cells (green) that produce mature sperm. No individual cross-section of the seminiferous tubule (dotted line) has all stages because of the wave of maturation along its length. (B) Molecular regulation of the murine mitosis/meiosis decision. Retinoic acid activates expression of STRA8, which governs meiotic entry by an unknown mechanism. NOS2 has been implicated in repression of the stra 8 mRNA as well as mRNAs encoding the meiotic machinery. In the male fetal gonad, Cyp26b1 inhibits retinoic acid and blocks meiotic entry. Solid arrows and lines indicate direct molecular regulation; dashed line indicates control that may be either direct or indirect. 
degradation of retinoic acid might prevent meiotic entry in the fetal testis (Fig. 4B). Subsequent experiments supported that model (Bowles et al. 2006; Koubova et al. 2006). For example, RA addition to a fetal male gonad induced early markers of meiosis, including STRA8, and suppressed the pluripotency marker Oct4, whereas RA antagonists had the opposite effect. Moreover, germ cells in the fetal testis entered the meiotic cell cycle in a Cyp26b1 mutant. Therefore, RA is capable of driving both female and male fetal germ cells into meiosis.

STRA8 is expressed specifically in meiotic germ cells in the fetal ovary and in the juvenile testis, suggesting that STRA8 might act downstream from RA to induce meiotic entry in both sexes (Fig. 4B) (Oulad-Abdelghani et al. 1996; Baltus et al. 2006; Anderson et al. 2008; Mark et al. 2008). Indeed, in stra8 null mutants, germ cells fail to enter the meiotic cell cycle, both in fetal female gonads and juvenile male gonads (Baltus et al. 2006; Anderson et al. 2008). An alternate view, that STRA8 instead functions in early meiotic prophase, comes from a parallel analysis of a stra 8 mutant (Mark et al. 2008). The likely resolution is that STRA8 governs both initiation into the meiotic cell cycle and early meiotic progression, with genetic modifiers generating the different phenotypes. The STRA8 "novel" amino acid sequence includes a putative helix-loop-helix domain (Baltus et al. 2006), and its subcellular localization encompasses both nucleus and cytoplasm (Oulad-Abdelghani et al. 1996; Tedesco et al. 2009). The idea that STRA8 may be a transcription factor has garnered some experimental support (Tedesco et al. 2009) but that conclusion is not yet confirmed. Most importantly, STRA8 is required for meiotic entry in both male and female germ cells, putting to rest the hypothesis that the mitosis/meiosis decision might be equivalent to the sperm/oocyte decision.

NOS2 and DAZ Also Influence the Fetal Mitosis/Meiosis Decision

Two conserved RNA binding proteins also influence the fetal mitosis/meiosis decision. The Nanos-related NOS2 protein prevents meiotic entry in fetal male germ cells and can be induced to do the same in fetal female germ cells when ectopically expressed (Fig. 4B) (Suzuki and Saga 2008). NOS2 is normally expressed in fetal male germ cells, just as Cy26b1 begins to decrease. In the absence of NOS2, male germ cells fail to maintain their $\mathrm{G}_{0} / \mathrm{G}_{1}$ cell cycle arrest and appear to embark on the meiotic cell cycle, but eventually die. However, in a double mutant that removes both NOS2 and the BAX2 proapoptotic regulator, fetal male germ cells expressed STRA8 aberrantly and entered the meiotic cell cycle (Suzuki and Saga 2008). Nanos proteins are translational repressors (Gray and Wickens 1998), and NOS2 itself interacts with the CCR4-Not deadenylation complex (Suzuki et al. 2010). Importantly, immunoprecipitation of a FLAGtagged NOS2 brings down stra 8 mRNA along with other transcripts implicated in meiosis (e.g., sycp3) (Suzuki et al. 2010). The likely scenario is that NOS2 represses both a regulator of meiotic entry (stra8) plus critical components of the meiotic machinery (Fig. 4B). In addition to NOS2, the DAZ (deleted in azoospermia) protein renders fetal germ cells of both sexes responsive to RA signaling (Lin et al. 2008). Therefore, both NOS2 and DAZ are capable of influencing meiotic entry in murine germ cells of either sex.

\section{PLANTS: GERMLINE/SOMA DECISION MEETS THE MITOSIS/MEIOSIS DECISION}

An embryonic germline lineage does not exist in plants. Instead, individual germ cells are induced from somatic tissues in the developing flower (Walbot and Evans 2003). Those germ cells, termed generically "sporocytes" in the two sexes or "megaspore mother cell" and "pollen mother cell" in females and males, respectively, typically enter into the meiotic cell cycle soon after their induction from the somatic hypodermal tissue. Therefore, meiotic entry is typically linked to germ cell specification in plants. However, in some mutant strains, germ cells can be specified and generate gametes without meiotic entry, a process termed apomixis (e.g., Ravi et al. 2008). The germline/soma and 
mitosis/meiosis decisions are therefore not inextricably linked.

Multiple regulators of germ cell specification and meiotic entry have been identified in Arabidopsis, maize, and rice (Ma 2005; Hamant et al. 2006; Mercier and Grelon 2008; Yang et al. 2010). The analyses of these regulators have provided important clues about controls of meiotic entry in plants. First, extrinsic signaling induces germ cell differentiation, including meiotic entry. In both the Arabidopsis dicot and rice monocot, a leucine-rich repeat receptor-like protein kinase controls germ cell induction (reviewed in Ma 2005), suggesting an ancient signaling mechanism among plants. Second, intrinsic regulators control meiotic entry or early meiotic prophase in both ovules and anthers (Motamayor et al. 2000; Mercier et al. 2003; Pawlowski et al. 2009). These include the Arabidopsis SWITCH1 and maize AMEIOTIC1 genes, which encode homologous plant-specific proteins that localize to the nucleus. SWITCH1 is required for the normal pattern of histone modifications, suggesting a role in chromatin modification (Boateng et al. 2008). Similarly, a germ cell specific Argonaute controls both early meiotic prophase and histone modifications in rice (Nonomura et al. 2007). Therefore, chromatin modification has been implicated as a likely molecular mechanism that influences meiotic entry in all three model plants.

\section{EMERGING THEMES}

Recent advances in understanding molecular controls of the mitosis/meiosis decision reveal several unifying themes. Some of those themes extend to yeast and plants, whereas others appear specific to metazoans. Rather than repeat references here, readers are referred for the most part to individual sections above for citations.

1. Extrinsic signals control the mitosis/meiosis decision. Extrinsic cues control the mitosis/ meiosis decision in S. cerevisiae and S. pombe (nutritional environment), C. elegans (Notch signaling), Drosophila (BMP signaling), and mice (retinoic acid signaling). In addition, signaling is responsible for germ cell induction in plants, which immediately precedes meiotic entry.

2. Intrinsic regulators of the mitosis/meiosis decision control the early meiotic program, often by regulation of mRNAs. Many of the key intrinsic regulators of the animal mitosis/meiosis decision regulate mRNAs: C. elegans FBF directly represses a battery of mRNAs encoding the meiotic program, Drosophila Bam/bgcn regulates expression of the nanos mRNA, and murine NOS2 RNAbinding protein appears to control the expression of mRNAs encoding STRA8 and a synaptonemal complex protein. The prevalence of RNA regulation is striking and extends to S. pombe, in which Mei2 and meiRNA antagonize the sequestration of meiotic mRNAs. By contrast, intrinsic regulators of the decision in plants have been implicated in chromatin regulation, which is reminiscent of the transcriptional activation of the early meiotic program in $S$. cerevisiae. The common thread is use of intrinsic regulators to govern the meiotic program.

3. Key regulators of meiotic entry are genderneutral. The mitosis/meiosis decision can differ in the two sexes in a variety of ways, including timing and position of meiotic entry. Yet the same central regulators control meiotic entry in both sexes in C. elegans, mice, plants, and probably Drosophila. Because the mitosis/ meiosis decision in C. elegans is not dramatically different in the two sexes, it was not surprising that gender-neutral regulators controlled the decision. However, in mice, where the decision is dramatically different in the two sexes, the discovery of regulators that control meiotic entry in both sexes was a real surprise and major breakthrough. The emerging theme is that gender-neutral regulators of the mitosis/meiosis decision can be modulated to drive meiotic entry in a sex-specific manner.

4. A close relationship exists between GSC selfrenewal and mitosis/meiosis controls in animals. GSC loss occurs aberrantly in C. elegans and Drosophila on removal of self-renewal regulators, and it occurs naturally in female 
mammals during fetal development. In all three cases, GSC loss is accompanied by the meiotic entry and differentiation of all germ cells. In C. elegans and Drosophila, molecular regulators of GSC self-renewal and meiotic entry are directly antagonistic. In mice, pluripotency factors (e.g., Oct4) are similarly antagonistic with RA signaling and STRA8, although the mechanism of that interaction is not yet known (Bowles and Koopman 2007).

5. Commitment to the meiotic cell cycle must be actively maintained. S. cerevisiae and C. elegans cells can enter the meiotic cell cycle and progress into pachytene, but then return to mitotic divisions, suggesting that commitment to the meiotic cell cycle must be actively maintained. In Drosophila, spermatogonial cells can dedifferentiate into GSCs (Sheng et al. 2009), suggesting a similar need for active maintenance of meiotic progression in flies. An irreversible commitment to meiosis only occurs at the meiosis I division in S. cerevisiae and may be similarly late in C. elegans.

\section{OPEN QUESTIONS}

The major advances made in understanding the mitosis/meiosis decision over the past decade have answered some key questions and opened the door to others.

1. What are the terminal regulators of the mitosis/meiosis decision in Drosophila, mice and plants? Terminal regulators act at the end of a pathway to execute the function of the pathway. The terminal regulators of the mitosis/meiosis pathway include the IME1 transcription factor in $S$. cerevisiae and the Mei2/MeiRNA regulators in S. pombe. In $C$. elegans, FBF responds to Notch signaling to directly repress the meiotic program, and GLD-1 directly represses cyclin E mRNA and the mitotic cell cycle. What are the terminal regulators in Drosophila, mice, and plants? Bam/bgcn, STRA8, and SWI1/AM1 are the best current candidates, but pathways must be filled out and direct links established with the meiotic program to be sure.
Regulation of the Mitosis/Meiosis Decision

2. Are regulators of the mitosis/meiosis decision conserved? Mitosis/meiosis regulators are not conserved among all eukaryotes (yeast to human), but the pathways in multicellular organisms are fragmentary and conservation of at least some key regulators remains a possibility. One mitosis/meiosis regulator that may be broadly conserved among metazoans is FBF, a $C$. elegans PUF RNA-binding protein. FBF regulates GSC maintenance in nematodes and directly represses the meiotic program as one part of this role. The Pumilio PUF RNA-binding protein is similarly required for GSC maintenance in Drosophila ovaries (Lin and Spradling 1997; Forbes and Lehmann 1998) and the Pum1 and Pum2 PUF RNAbinding proteins are enriched in GSCs in mice and humans (Moore et al. 2003). However, a role for these fly and mammalian PUF counterparts in the mitosis/meiosis decision remains an open question.

3. How does mitosis/meiosis regulation interface with controls of other germ cell fates? Research during the past few years has clarified relationships between the mitosis/ meiosis decision and the decisions of selfrenewal versus differentiation and male versus female, at least in nematodes and mice, respectively. An open question is how universal the answers are. For example, do GSC regulators control both the meiotic program and general differentiation regulators in all metazoans or indeed in all multicellular organisms? And what about regulatory relationships with other decisions (e.g., proliferation versus self-renewal and maintenance, germline survival versus apoptosis) and other more global events (e.g., aging, starvation)?

4. Do nutritional cues affect the mitosis/ meiosis decision in animal germ cells? Nutritional cues are central to the mitosis/meiosis decision in S. cerevisiae and S. pombe, but do they play a similar role in other creatures? In male mice, vitamin A depletion (VAD) yields germlines with only GSCs or early differentiating premeiotic germ cells (van Pelt and de Rooij 1990); an attractive hypothesis 
J. Kimble

is that VAD blocks meiotic entry (Bowles and Koopman 2007; Clagett-Dame and Knutson 2011). In nematodes, starvation has a similar effect, which is dependent on a nuclear hormone receptor, NHR-49, that controls fatty acid metabolism in response to starvation (Van Gilst et al. 2005; Angelo and Van Gilst 2009). In flies, nutrition affects the rate of mitotic proliferation (Hsu et al. 2008), but its effect on the mitotic/meiotic decision has not yet been reported. The broader role of nutrition on the mitosis/meiosis decision in multicellular creatures is virtually virgin territory for future exploration.

5. What is the clinical impact of understanding the mitosis/meiosis decision? Understanding the mitosis/meiosis decision has clear implications for human fertility and germ cell tumors. For example, in adult men, the mitotic and meiotic cell cycles are normally kept in a tightly controlled balance between spermatogonial stem cells (SSCs) and maturing spermatocytes. If that balance is tipped aberrantly toward meiotic entry, SSCs are depleted, and if tipped toward mitotic divisions, uncontrolled germ cell proliferation and perhaps cancer result. An in depth understanding of the molecular mechanisms that modulate the balance will therefore be crucial for developing therapies that impact human reproduction and health. One possible example might be the development of a male contraceptive that is based on the inhibition of molecular events specific to the mitosis/ meiosis decision. Vitamin A depletion renders males sterile and its effects can be reversed-but Vitamin A depletion affects many tissues deleteriously. The generation of a drug that targets a germ cell-specific component of the pathway (e.g., STRA8) might be equally effective but have few side effects.

\section{CONCLUSIONS AND PERSPECTIVES}

Our understanding of the mitosis/meiosis decision in germ cells of multicellular organisms has been transformed during the past 5 years. One conceptual breakthrough is that mitosis/meiosis regulators are gender-neutralthey control meiotic entry in the germ cells of both sexes. This basic idea holds true in nematodes, mice, plants, and probably flies. The dramatic sex-specific timing of the mammalian mitosis/meiosis decision, which historically was equated with the sperm/oocyte decision, actually relies on sex-specific regulation of the gender-neutral mitosis/meiosis regulators. A second conceptual breakthrough is that regulators of germline stem cells control entry into the meiotic cell cycle as part of their larger program to maintain the stem cells in a naive, undifferentiated state. Plants do not have germline stem cells, per se, but instead possess totipotent somatic cells that may play a similar but plantspecific role. The relationship between stem cell and mitosis/meiosis regulators may therefore be specialized for animal germ cells.

The decision of a germ cell to progress through either the mitotic or the meiotic cell cycle is an ancient one with important implications for reproduction and health in humans and improved methods of agriculture in plants. Although breakthroughs have been made, our molecular understanding of the mitosis/meiosis decision remains in its infancy. The next decade will expand our knowledge of the mitosis / meiosis molecular circuitry in multiple organisms and clarify the relationship of that circuitry with GSC controls, totipotency controls, and the sperm/oocyte decision.

\section{ACKNOWLEDGMENTS}

I thank Kathy Barton, Sarah Crittenden, Matt Evans, Clint Morgan, and Dave Zarkower for comments, and Anne Helsley and Laura Vanderploeg for assistance in preparation of the manuscript and figures. J.K. is an investigator of the Howard Hughes Medical Institute and is supported by the NIH.

\section{REFERENCES}

Anderson EL, Baltus AE, Roepers-Gajadien HL, Hassold TJ, de Rooij DG, van Pelt AMM, Page DC. 2008. Stra8 and its inducer, retinoic acid, regulate meiotic initiation in both spermatogenesis and oogenesis in mice. Proc Natl Acad Sci 105: 14976-14980. 
Angelo G, Van Gilst M. 2009. Starvation protects germline stem cells and extends reproductive longevity in $C$. elegans. Science 326: 954-958.

Ariz M, Mainpal R, Subramaniam K. 2009. C. elegans RNAbinding proteins PUF-8 and MEX-3 function redundantly to promote germline stem cell mitosis. Dev Biol 326: 295-304.

Baltus AE, Menke DB, Hu YC, Goodheart ML, Carpenter AE, de Rooij DG, Page DC. 2006. In germ cells of mouse embryonic ovaries, the decision to enter meiosis precedes premeiotic DNA replication. Nat Genet 38: 1430-1434.

Barton MK, Kimble J. 1990. fog-1, a regulatory gene required for specification of spermatogenesis in the germ line of Caenorhabditis elegans. Genetics 125: 29-39.

Belfiore M, Pugnale P, Saudan Z, Puoti A. 2004. Roles of the C. elegans cyclophilin-like protein MOG-6 in MEP-1 binding and germline fates. Development 131: 29352945.

Berset T, Fröhli Hoier E, Battu G, Canevascini S, Hajnal A. 2001. Notch inhibition of RAS signaling through MAP kinase phosphatase LIP-1 during C. elegans vulval development. Science 291: 1055-1058.

Biedermann B, Wright J, Senften M, Kalchhauser I, Sarathy G, Lee MH, Ciosk R. 2009. Translational repression of cyclin $\mathrm{E}$ prevents precocious mitosis and embryonic gene activation during C. elegans meiosis. Dev Cell 17: 355-364.

Boateng KA, Yang X, Dong F, Owen HA, Makaroff CA. 2008. SWI1 is required for meiotic chromosome remodeling events. Mol Plant 1: 620-633.

Bowles J, Koopman P. 2007. Retinoic acid, meiosis and germ cell fate in mammals. Development 134: 3401-3411.

Bowles J, Knight D, Smith C, Wilhelm D, Richman J, Mamiya S, Yashiro K, Chawengsaksophak K, Wilson MJ, Rossant J, et al. 2006. Retinoid signaling determines germ cell fate in mice. Science 312: 596-600.

Chen D, McKearin DM. 2003. A discrete transcriptional silencer in the bam gene determines asymmetric division of the Drosophila germline stem cell. Development 130: 1159-1170.

Cinquin O, Crittenden SL, Morgan DE, Kimble J. 2010. Progression from a stem cell-like state to early differentiation in the C. elegans germ line. Proc Natl Acad Sci 107: 2048-2053.

Ciosk R, DePalma M, Priess JR. 2004. ATX-2, the C. elegans ortholog of ataxin 2, functions in translational regulation in the germline. Development 131: 4831-4841.

Ciosk R, DePalma M, Priess JR. 2006. Translational regulators maintain totipotency in the Caenorhabditis elegans germline. Science 311: 851-853.

Clagett-Dame M, Knutson D. 2011. Vitamin A in reproduction and development. Nutrients 3: 395-428.

Cox DN, Chao A, Baker J, Chang L, Qiao D, Lin H. 1998. A novel class of evolutionarily conserved genes defined by piwi are essential for stem cell self-renewal. Genes Dev 12: $3715-3727$.

Crittenden SL, Bernstein DS, Bachorik JL, Thompson BE, Gallegos M, Petcherski AG, Moulder G, Barstead R, Wickens M, Kimble J. 2002. A conserved RNA-binding protein controls germline stem cells in Caenorhabditis elegans. Nature 417: 660-663.
Crittenden SL, Leonhard KA, Byrd DT, Kimble J. 2006. Cellular analyses of the mitotic region in the Caenorhabditis elegans adult germ line. Mol Biol Cell 17: 3051-3061.

Davies EL, Fuller MT. 2008. Regulation of self-renewal and differentiation in adult stem cell lineages: Lessons from the Drosophila male germ line. Cold Spring Harbor Symp Quant Biol 73: 137-145.

Eckmann CR, Kraemer B, Wickens M, Kimble J. 2002. GLD-3, a Bicaudal-C homolog that inhibits FBF to control germline sex determination in C. elegans. Dev Cell 3: 697-710.

Eckmann CR, Crittenden SL, Suh N, Kimble J. 2004. GLD-3 and control of the mitosis/meiosis decision in the germline of Caenorhabditis elegans. Genetics 168: 147-160.

Forbes A, Lehmann R. 1998. Nanos and Pumilio have critical roles in the development and function of Drosophila germline stem cells. Development 125: 679-690.

Forsburg SL. 2002. Only connect: Linking meiotic DNA replication to chromosome dynamics. Mol Cell 9: 703-711.

Francis R, Barton MK, Kimble J, Schedl T. 1995. gld-1, a tumor suppressor gene required for oocyte development in Caenorhabditis elegans. Genetics 139: 579-606.

Gönczy P, Matunis E, DiNardo S. 1997. bag-of-marbles and benign gonial cell neoplasm act in the germline to restrict proliferation during Drosophila spermatogenesis. Development 124: 4361-4371.

Gray NK, Wickens M. 1998. Control of translation initiation in animals. Annu Rev Cell Dev Biol 14: 399-458.

Hamant O, Ma H, Cande WZ. 2006. Genetics of meiotic prophase I in plants. Annu Rev Plant Biol 57: 267-302.

Hansen D, Wilson-Berry L, Dang T, Schedl T. 2004. Control of the proliferation versus meiotic development decision in the C. elegans germline through regulation of GLD-1 protein accumulation. Development 131: 93-104.

Hansen M, Hsu A-L, Dillin A, Kenyon C. 2005. New genes tied to endocrine, metabolic, and dietary regulation of lifespan from a Caenorhabditis elegans genomic RNAi screen. PLoS Genet 1: 119-128.

Harigaya Y, Yamamoto M. 2007. Molecular mechanisms underlying the mitosis/meiosis decision. Chromosome Res 15: 523-537.

Harigaya Y, Tanaka H, Yamanaka S, Tanaka K, Watanabe Y, Tsutsumi C, Chikashige Y, Hiraoka Y, Yamashita A, Yamamoto M. 2006. Selective elimination of messenger RNA prevents an incidence of untimely meiosis. Nature 442: $45-50$.

Hasegawa E, Karashima T, Sumiyoshi E, Yamamoto M. 2006. C. elegans CPB-3 interacts with DAZ-1 and functions in multiple steps of germline development. Dev Biol 295: 689-699.

Hsu HJ, LaFever L, Drummond-Barbosa D. 2008. Diet controls normal and tumorous germline stem cells via insulin-dependent and -independent mechanisms in Drosophila. Dev Biol 313: 700-712.

Insco ML, Leon A, Tam CH, McKearin DM, Fuller MT. 2009. Accumulation of a differentiation regulator specifies transit amplifying division number in an adult stem cell lineage. Proc Natl Acad Sci 106: 22311-22316.

Kadyk LC, Kimble J. 1998. Genetic regulation of entry into meiosis in Caenorhabditis elegans. Development 125: $1803-1813$. 
J. Kimble

Kassir Y, Adir N, Boger-Nadjar E, Guttmann Raviv N, Rubin-Bejerano I, Sagee S, Shenhar G. 2003. Transcriptional regulation of meiosis in budding yeast. Int Rev Cytol 224: 111-171.

Kawase E, Wong MD, Ding BC, Xie T. 2004. Gbb/Bmp signaling is essential for maintaining germline stem cells and for repressing bam transcription in the Drosophila testis. Development 131: 1365-1375.

Kerins JA, Hanazawa M, Dorsett M, Schedl T. 2010. PRP-17 and the pre-mRNA splicing pathway are preferentially required for the proliferation versus meiotic development decision and germline sex determination in Caenorhabditis elegans. Dev Dyn 239: 1555-1572.

Kershner AM, Kimble J. 2010. Genome-wide analysis of mRNA targets for Caenorhabditis elegans FBF, a conserved stem cell regulator. Proc Natl Acad Sci 107: 3936-3941.

Kim JY, Lee YC, Kim C. 2010. Direct inhibition of Pumilo activity by Bam and Bgcn in Drosophila germ line stem cell differentiation. J Biol Chem 285: 4741-4746.

Kimble J, Crittenden SL. 2005. Germline proliferation and its control. In WormBook (ed. The C. elegans Research Community), 10.1895/wormbook.1.13.1. www. wormbook.org.

Kimble J, Crittenden SL. 2007. Controls of germline stem cells, entry into meiosis, and the sperm/oocyte decision in Caenorhabditis elegans. Annu Rev Cell Dev Biol 23: 405-433.

Kimble J, Page DC. 2007. The mysteries of sexual identity. The germ cell's perspective. Science 316: 400-401.

Kimble JE, White JG. 1981. On the control of germ cell development in Caenorhabditis elegans. Dev Biol 81: 208-219.

Koubova J, Menke DB, Zhou Q, Capel B, Griswold MD, Page DC. 2006. Retinoic acid regulates sex-specific timing of meiotic initiation in mice. Proc Natl Acad Sci 103: 2474-2479.

Lamont LB, Crittenden SL, Bernstein D, Wickens M, Kimble J. 2004. FBF-1 and FBF-2 regulate the size of the mitotic region in the C. elegans germline. Dev Cell 7: 697-707.

Lee M-H, Schedl T. 2010. C. elegans STAR proteins, GLD-1 and ASD-2, regulate specific RNA targets to control development. In Posttranscriptional regulation by STAR proteins: Control of RNA metabolism in development and disease (ed. Volk T, Atrzt K), pp. 106-122. Landes Bioscience and Springer SBM, New York.

Lee M-H, Hook B, Lamont LB, Wickens M, Kimble J. 2006. LIP-1 phosphatase controls the extent of germline proliferation in Caenorhabditis elegans. EMBO J 25: 88-96.

Lee M-H, Hook B, Pan G, Kershner AM, Merritt C, Seydoux G, Thomson JA, Wickens M, Kimble J. 2007a. Conserved regulation of MAP kinase expression by PUF RNAbinding proteins. PLoS Genet 3: e233.

Lee M-H, Ohmachi M, Arur S, Nayak S, Francis R, Church D, Lambie E, Schedl T. 2007b. Multiple functions and dynamic activation of MPK-1 extracellular signalregulated kinase signaling in Caenorhabditis elegans germline development. Genetics 177: 2039-2062.

Li Y, Minor NT, Park JK, McKearin DM, Maines JZ. 2009. Bam and Bgen antagonize Nanos-dependent germ-line stem cell maintenance. Proc Natl Acad Sci 106: 93049309 .

Lin H, Spradling AC. 1997. A novel group of pumilio mutations affects the asymmetric division of germline stem cells in the Drosophila ovary. Development 124: 2463 2476.

Lin Y, Gill ME, Koubova J, Page DC. 2008. Germ cell-intrinsic and -extrinsic factors govern meiotic initiation in mouse embryos. Science 322: 1685-1687.

Ma H. 2005. Molecular genetic analyses of microsporogenesis and microgametogenesis in flowering plants. Annu Rev Plant Biol 56: 393-434.

Maine EM, Hansen D, Springer D, Vought VE. 2004. Caenorhabditis elegans atx-2 promotes germline proliferation and the oocyte fate. Genetics 168: 817-830.

Marin VA, Evans TC. 2003. Translational repression of a C. elegans Notch mRNA by the STAR/KH domain protein GLD-1. Development 130: 2623-2632.

Mark M, Jacobs H, Oulad-Abdelghani M, Dennefeld C, Féret B, Vernet N, Codreanu C-A, Chambon P, Ghyselinck NB. 2008. STRA8-deficient spermatocytes initiate, but fail to complete, meiosis and undergo premature chromosome condensation. J Cell Sci 121: 3233-3242.

McKearin DM, Spradling AC. 1990. bag-of-marbles: A Drosophila gene required to initiate both male and female gametogenesis. Genes Dev 4: 2242-2251.

Mercier R, Grelon M. 2008. Meiosis in plants: Ten years of gene discovery. Cytogenet Genome Res 120: 281-290.

Mercier R, Armstrong SJ, Horlow C, Jackson NP, Makaroff CA, Vezon D, Pelletier G, Jones GH, Franklin FC. 2003. The meiotic protein SWI1 is required for axial element formation and recombination initiation in Arabidopsis. Development 130: 3309-3318.

Merritt C, Seydoux G. 2010. Transgenic solutions for the germline. In WormBook (ed. The C. elegans Research Community), 10.1895/wormbook.1.148.1. www. wormbook.org.

Merritt C, Rasoloson D, Ko D, Seydoux G. 2008. 3' UTRs are the primary regulators of gene expression in the C. elegans germline. Curr Biol 18: 1476-1482.

Michaelson D, Korta DZ, Capua Y, Hubbard EJ. 2010. Insulin signaling promotes germline proliferation in C. elegans. Development 137: 671-680.

Mitchell AP. 1994. Control of meiotic gene expression in Saccharomyces cerevisiae. Microbiol Rev 58: 56-70.

Moore FL, Jaruzelska J, Fox MS, Urano J, Firpo MT, Turek PJ, Dorfman DM, Reijo Pera RA. 2003. Human Pumilio-2 is expressed in embryonic stem cells and germ cells and interacts with DAZ (Deleted in AZoospermia) and DAZ-like proteins. Proc Natl Acad Sci 100: 538-543.

Morgan DE, Crittenden SL, Kimble J. 2010. The C. elegans adult male germline: Stem cells and sexual dimorphism. Dev Biol 346: 204-214.

Motamayor JC, Vezon D, Bajon C, Sauvanet A, Grandjean O, Marchand M, Bechtold N, Pelletier G, Horlow C. 2000. Switch (swi1), and Arabidopsis thaliana mutant affected in the female meiotic switch. Sex Plant Reprod 12: $209-218$.

Nonomura K, Morohoshi A, Nakano M, Eiguchi M, Miyao A, Hirochika H, Kurata N. 2007. A germ cell specific gene of the ARGONAUTE family is essential for the 
progression of premeiotic mitosis and meiosis during sporogenesis in rice. Plant Cell 19: 2583-2594.

Ohlstein B, McKearin D. 1997. Ectopic expression of the Drosophila Bam protein eliminates oogenic germline stem cells. Development 124: 3651-3662.

Ohlstein B, Lavoie CA, Vef O, Gateff E, McKearin DM. 2000. The Drosophila cystoblast differentiation factor, benign gonial cell neoplasm, is related to DExH-box proteins and interacts genetically with bag-of-marbles. Genetics 155: $1809-1819$.

Oulad-Abdelghani M, Bouillet P, Decimo D, Gansmuller A, Heyberger S, Dolle P, Bronner S, Lutz Y, Chambon P. 1996. Characterization of a premeiotic germ cell-specific cytoplasmic protein encoded by Stra8, a novel retinoic acid-responsive gene. J Cell Biol 135: 469-477.

Pawlowski WP, Wang CJ, Golubovskaya IN, Szymaniak JM, Shi L, Hamant O, Zhu T, Harper L, Sheridan WF, Cande WZ. 2009. Maize AMEIOTIC1 is essential for multiple early meiotic processes and likely required for the initiation of meiosis. Proc Natl Acad Sci 106: 3603-3608.

Petcherski AG, Kimble J. 2000. LAG-3 is a putative transcriptional activator in the C. elegans Notch pathway. Nature 405: 364-368.

Ravi M, Marimuthu MP, Siddiqi I. 2008. Gamete formation without meiosis in Arabidopsis. Nature 451: 1121-1124.

Shen R, Weng C, Yu J, Xie T. 2009. eIF4A controls germline stem cell self-renewal by directly inhibiting BAM function in the Drosophila ovary. Proc Natl Acad Sci 106: $11623-11628$.

Sheng XR, Brawley CM, Matunis EL. 2009. Dedifferentiating spermatogonia outcompete somatic stem cells for niche occupancy in the Drosophila testis. Cell Stem Cell 5: 191-203.

Simchen G. 2009. Commitment to meiosis: What determines the mode of division in budding yeast? BioEssays 31: $169-177$.

Smardon A, Spoerke JM, Stacey SC, Klein ME, Mackin N, Maine EM. 2000. EGO-1 is related to RNA-directed RNA polymerase and functions in germ-line development and RNA interference in C. elegans. Curr Biol 10: 169-178.

Subramaniam K, Seydoux G. 2003. Dedifferentiation of primary spermatocytes into germ cell tumors in C. elegans lacking the Pumilio-like protein PUF-8. Curr Biol 13: $134-139$.

Suh N, Jedamzik B, Eckmann CR, Wickens M, Kimble J. 2006. The GLD-2 poly(A) polymerase activates gld-1 mRNA in the Caenorhabditis elegans germ line. Proc Natl Acad Sci 103: 15108-15112.

Sundaram MV. 2006. RTK/Ras/MAPK signaling. In WormBook (ed. The C. elegans Research Community), 10.1895/wormbook.1.80.1. www.wormbook.org.
Suzuki A, Saga Y. 2008. Nanos2 suppresses meiosis and promotes male germ cell differentiation. Genes Dev 22: $430-435$.

Suzuki H, Saba R, Sada A, Saga Y. 2010. The Nanos3-3'UTR is required for germ cell specific NANOS3 expression in mouse embryos. PLoS ONE 5: e9300.

Tedesco M, La Sala G, Barbagallo F, De Felici M, Farini D. 2009. STRA8 shuttles between nucleus and cytoplasm and displays transcriptional activity. J Biol Chem 284: 35781-35793.

Thompson BE, Bernstein DS, Bachorik JL, Petcherski AG, Wickens M, Kimble J. 2005. Dose-dependent control of proliferation and sperm specification by FOG-1/CPEB. Development 132: 3471-3481.

Thompson BE, Lamont LB, Kimble J. 2006. Germ-line induction of the Caenorhabditis elegans vulva. Proc Natl Acad Sci 103: 620-625.

van den Heuvel S. 2005. Cell-cycle regulation. In WormBook (ed. The C. elegans Research Community), 10.1895/ wormbook.1.28.1. www.wormbook.org.

Van Gilst MR, Hadjivassiliou H, Yamamoto KR. 2005. A Caenorhabditis elegans nutrient response system partially dependent on nuclear receptor NHR-49. Proc Natl Acad Sci 102: 13496-13501.

van Pelt AM, de Rooij DG. 1990. Synchronization of the seminiferous epithelium after vitamin A replacement in vitamin A-deficient mice. Biol Reprod 43: 363-367.

Vershon AK, Pierce M. 2000. Transcriptional regulation of meiosis in yeast. Curr Opin Cell Biol 12: 334-339.

Walbot V, Evans MM. 2003. Unique features of the plant life cycle and their consequences. Nat Rev Genet 4: 369-379.

Wang L, Eckmann CR, Kadyk LC, Wickens M, Kimble J. 2002. A regulatory cytoplasmic poly(A) polymerase in Caenorhabditis elegans. Nature 419: 312-316.

Watanabe Y, Yamamoto M. 1994. S. pombe mei2 ${ }^{+}$encodes an RNA-binding protein essential for premeiotic DNA synthesis and meiosis I, which cooperates with a novel RNA species meiRNA. Cell 78: 487-498.

Watanabe Y, Yokobayashi S, Yamamoto M, Nurse P. 2001. Pre-meiotic $S$ phase is linked to reductional chromosome segregation and recombination. Nature 409: 359-363.

Yamamoto M. 1996. The molecular control mechanisms of meiosis in fission yeast. Trends Biochem Sci 21: 18-22.

Yang WC, Shi DQ, Chen YH. 2010. Female gametophyte development in flowering plants. Annu Rev Plant Biol 61: $89-108$

Zhang N, Xie T. 2009. Extrinsic and intrinsic control of germline stem cell regulation in the Drosophila ovary. In Regulatory networks in stem cells (ed. Rajasekhar VK, Vemuri MC), pp. 155-164. Humana Press, New York. 


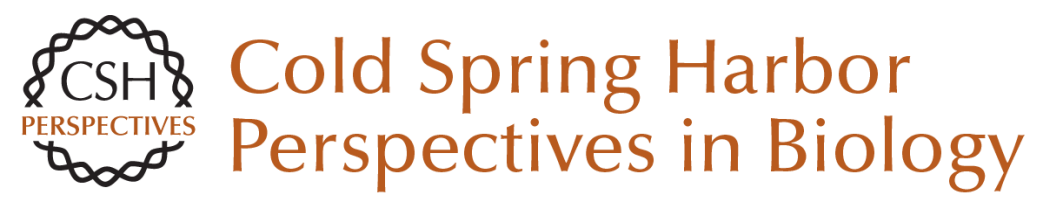

\section{Molecular Regulation of the Mitosis/Meiosis Decision in Multicellular Organisms}

Judith Kimble

Cold Spring Harb Perspect Biol 2011; doi: 10.1101/cshperspect.a002683 originally published online June 6,2011

\section{Subject Collection Germ Cells}

RNA Granules in Germ Cells

Ekaterina Voronina, Geraldine Seydoux, Paolo

Sassone-Corsi, et al.

Function of the Sex Chromosomes in Mammalian

Fertility

Edith Heard and James Turner

Small Noncoding RNAs in the Germline Jonathan P. Saxe and Haifan Lin

Mammalian Genomic Imprinting

Marisa S. Bartolomei and Anne C. Ferguson-Smith

Molecular Regulation of the Mitosis/Meiosis

Decision in Multicellular Organisms Judith Kimble

Selection in the Rapid Evolution of Gamete

Recognition Proteins in Marine Invertebrates Victor D. Vacquier and Willie J. Swanson
Developmental Control of Oocyte Maturation and

Egg Activation in Metazoan Models Jessica R. Von Stetina and Terry L. Orr-Weaver

Translational Control in Oocyte Development Joel D. Richter and Paul Lasko

Unique Aspects of Transcription Regulation in Male Germ Cells Helen White-Cooper and Irwin Davidson

Germ Cell Intercellular Bridges Michael P. Greenbaum, Tokuko Iwamori, Gregory M. Buchold, et al.

Germline Stem Cells

Allan Spradling, Margaret T. Fuller, Robert E. Braun, et al.

Nuclear Transfer to Eggs and Oocytes J. B. Gurdon and lan Wilmut

For additional articles in this collection, see http://cshperspectives.cshlp.org/cgi/collection/

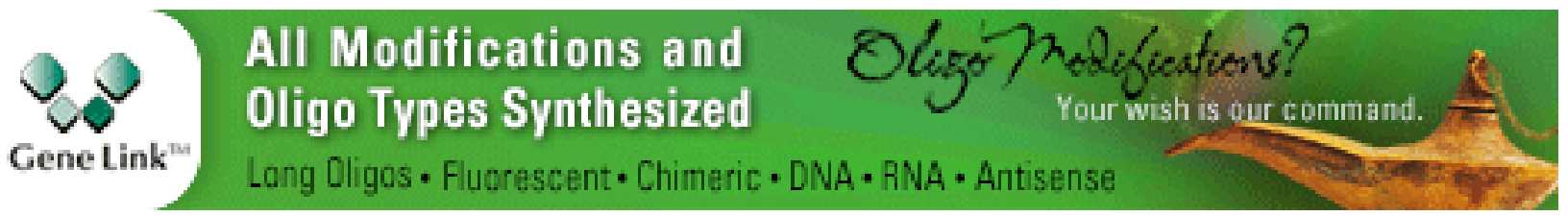

Copyright @ 2011 Cold Spring Harbor Laboratory Press; all rights reserved 